\title{
RADIO SOURCE COUNTS AT ULTRA LOW FREQUENCIES: RESULTS, SIGNIFICANCE AND IMPLICATION FOR OBSERVATIONAL COSMOLOGY
}

\author{
K.P. SOKOLOV \\ Institute of Radio Astronomy, 4, Krasnoznamennaya Str., \\ Kharkov 310002, Ukraine
}

Analysis of cosmological evolution effects in the low-frequency source counts at $25 \mathrm{MHz}$ obtained with the UTR-2 radio telescope and conclusions on the large-scale extragalactic radio source space distribution are presented. The data require the existence of a decrease in the space distribution of the most distant extended radio sources.

The astrophysical value for radio cosmology of studying statistical properties of old extended extragalactic radio sources with low surface brightness and steep spectra which constitute the dominant population at ultra low frequencies $(<100 \mathrm{MHz})$ is considered. It is shown that these sources form one of the most significant class of objects for radio cosmology because:

1) the effects of extragalactic radio source cosmological evolution and general properties of their space distribution manifest themselves particularly strongly in samples of old extended sources;

2) these sources have a radiating lifetime which is comparable with the age of the Universe;

3) these objects are characterized by the least scatter in their intrinsic luminosities;

4) these objects form a subsample of an isotropically radiating "parent population" of radio sources which is not biased by alignment effects;

5) the conditions for the existence of a correlation between radio source flux densities and distances, which constitutes a basis for every source count analysis, are best satisfied for radio sources observed at ultra low frequencies. 